\title{
The Economic Burden of Cancers Attributable to Metabolic Syndrome in Korea
}

\author{
Dongwoo Kim ', Seok-Jun Yoon', Young-Hoon Gong ${ }^{1}$, Young Ae Kim², Hye-Young Seo ${ }^{3}$, Jihyun Yoon ${ }^{3}$, A-Rim Kim ${ }^{3}$ \\ ${ }^{1}$ Department of Preventive Medicine, Korea University College of Medicine, Seoul; ${ }^{2}$ Cancer Policy Branch, National Cancer Control Institute, National \\ Cancer Center, Goyang; ${ }^{3}$ Department of Public Health, Graduate School of Korea University, Seoul, Korea
}

Objectives: Metabolic syndrome is an important etiologic factor in the development of certain types of cancers. The economic cost of the treatment of cancer has been steadily increasing. We therefore estimated the economic burden of cancers attributable to metabolic syndrome in Korea.

Methods: We reviewed metabolic syndrome-related cancers and relative risk and then calculated population attributable fractions. We analyzed insurance claims data for metabolic syndrome-related cancers in 2012 in order to estimate the direct costs associated with these cancers, including hospitalization, outpatient visits, transportation costs, and caregivers' costs as well as indirect costs such as loss of productivity due to cancer treatment and premature death.

Results: In 2012, 18070 patients in Korea had cancers attributable to metabolic syndrome. The economic burden was USD 199.8 million and the direct and indirect costs were USD 124.5 million and USD 75.3 million, respectively.

Conclusions: We estimated the economic burden of cancers attributable to metabolic syndrome in Korea and the efforts are necessary to reduce this burden.

Key words: Metabolic syndrome, Neoplasm, Economic burden

\section{INTRODUCTION}

Metabolic syndrome (MS) is an overall concept that includes diabetes, hypertension, obesity, and dyslipidemia. In early days, the insulin resistance was a key factor in MS, diagnostic criteria were proposed in 2001 by the National Cholesterol Education Program Adult Treatment Panel III and the harmonizing definition was presented in 2009. The prevalence of MS is increasing

Received: March 31, 2015 Accepted: July 8, 2015

Corresponding author: Seok-Jun Yoon, MD, PhD

73 Inchon-ro, Seongbuk-gu, Seoul 136-705, Korea

Tel: +82-2-2286-1412, Fax: +82-2-927-7220

E-mail: yoonsj02@korea.ac.kr

This is an Open Access article distributed under the terms of the Creative Commons Attribution Non-Commercial License (http://creativecommons.org/licenses/bync/3.0/) which permits unrestricted non-commercial use, distribution, and reproduction in any medium, provided the original work is properly cited. steadily worldwide, and Korea is not an exception, as the prevalence of MS in Korea increased from $24.9 \%$ in 1998 to $32.4 \%$ in $2009[1,2]$.

MS is a well-known risk factor for cardiovascular disease, but some biological studies have suggested that it may be an important etiologic factor for the development of certain types of cancer [3]. A positive association between colon cancer and obesity, which is a component of MS, has been well established and the effects of each component of MS on cancer occurrence have been reported. However, few studies have assessed MS itself as a risk factor of cancer, because the diagnostic criteria of MS have been modified several times and may be applied differently depending on the country or regions. Nevertheless, a few large-scaled cohort studies have supported positive associations between the MS and some cancers [4] and several meta-analyses dealing with MS and cancer risk have been published $[5,6]$. 
The occurrence of cancer is increasing steadily in Korea, 1.3 million people were registered as patients with cancer in 2012 [7], the number of deaths from cancer in 2012 was 73 759, and the death rate from cancer (per 100000 population) in 2012 was 146.5 [8]. The economic cost of cancer treatment has steadily increased, and the health insurance benefit amount of the National Health Insurance Service of Korea was USD 3.6 billion in 2012 [7].

The effects of obesity and fasting glucose on cancer occurrence have been reported, and a population attributable fraction (PAF) for obesity has been calculated [9]. However the impact of MS on cancer has not been measured so we reviewed articles about the effect of MS on cancer occurrence and estimated the PAF of MS.

Several studies assessing the economic burden of cancer in Korea have been published $[10,11]$ but these studies have either estimated the economic cost of a single type of cancer or of cancer in general, meaning that the economic burden of cancers attributable to MS has not been studied. So we estimated the economic burden of MS-related cancers in Korea and the economic burden of cancers attributable to MS by using the PAF.

\section{METHODS}

\section{Selection of Metabolic Syndrome-related Cancers and Relative Risk}

Some studies have been published dealing with the association between MS as a whole and cancer, instead of focusing on isolated components of MS, such as obesity, hypertension, and fasting glucose levels. We investigated systematic reviews with meta-analysis about MS-related cancers. MS was associated with an increased risk of colorectal cancer in men (relative risk [RR], 1.33; 95\% confidence interval [Cl], 1.18 to 1.50 ) and women (RR, $1.41 ; 95 \% \mathrm{Cl}, 1.18$ to 1.70 ) [6]. In men, MS was associated with an increased risk of liver cancer (RR, 1.43; $95 \% \mathrm{Cl}, 1.23$ to 1.65 ) and bladder cancer (RR, $1.10 ; 95 \% \mathrm{Cl}, 1.02$ to 1.18$)$ [5]. In women, MS was associated with an increased risk of pancreatic cancer (RR, 1.58; 95\% Cl, 1.35 to 1.84 ), postmenopausal (PM) breast cancer (RR, 1.56; $95 \% \mathrm{Cl}, 1.08$ to 2.24 ), uterine corpus (RR, 1.61; $95 \% \mathrm{Cl}, 1.20$ to 2.15$)$ and ovarian cancer (RR, 1.26; $95 \% \mathrm{Cl}, 1.00$ to 1.59 ) [5]. The association with ovarian cancer was of borderline significance.

MS was not associated with a significantly elevated risk of premenopausal breast cancer [5], kidney cancer [12], cervical cancer [13], skin cancer [14], prostate cancer, thyroid cancer, or bladder cancer in women [5]. These cancers were excluded.

\section{Calculation of the Population Attributable}

Fraction and Prevalence of Metabolic Syndrome

We used the PAF of MS to calculate the economic burden of cancers attributable to MS. The PAF of MS is calculated using the following formula [15]:

$$
P A F=P \times(R R-1) /[P \times(R R-1)+1]
$$

where $P$ is the prevalence of exposure to the risk factor (MS) in the total population and $R R$ is the relative risk of MS for a specific cancer.

The prevalence of MS was determined to be $22.4 \%$ among men and $27.9 \%$ among women, based on the results of the 1998 Korean National Health and Nutrition Examination Survey (KNHANES) [1], which were chosen due to the time lag associated with the occurrence of cancer [9]. In the KNHANES, the diagnostic criteria of MS followed the National Cholesterol Education Program Adult Treatment Panel III, using the Asia-Pacific criteria for abdominal obesity ( $>90 \mathrm{~cm}$ for men; $>80 \mathrm{~cm}$ for women). Moreover, we applied the prevalence of MS in the 2001 KNHANES (26.9\% among men, 31.8\% among women) and the 2005 KNHANES (31.7\% among men, 29.5\% among women) for sensitivity analysis [1].

The PAFs of MS for colorectal cancer in men and women were $6.88 \%$ and $10.26 \%$. The PAFs of MS for pancreatic cancer, postmenopausal breast cancer, uterine corpus cancer and ovarian cancer in women was $13.93 \%, 13.51 \%, 14.54 \%$, and $6.76 \%$, respectively. The PAF of MS for bladder cancer in men was $2.19 \%$. Meanwhile, the proportion of cases of liver cancer attributable to infectious agents such as the hepatitis B virus or the hepatitis C virus was very high (84\%) in Korea [16], so the PAF for liver cancer was modified by multiplying it by the proportion of liver cancer not attributable to infectious agents. The PAF of MS for liver cancer in men was $1.47 \%$.

\section{Estimation of the Economic Burden}

The economic burden of disease can include direct costs, indirect costs, and intangible costs [17]. Intangible costs, such as pain and suffering or bereavement are omitted in most studies on the costs of disease because it is difficult to quantify these costs in monetary terms. Therefore these costs were not included in this study. Direct costs consist of medical costs including hospitalization, outpatient visits, and medication for the pur- 
pose of cancer treatment, as well as non-medical costs, including the cost of transportation and caregivers' costs. Indirect costs include loss of productivity associated with hospital admissions or outpatient visits in addition to costs related to premature death. The economic burden of cancers attributable to MS was estimated using the total costs of each cancer and PAF in the following equation:

$$
\begin{aligned}
& \sum S_{i j} \times P A F_{i j} \\
& i=\text { metabolic syndrome-related cancer } \\
& j=\text { sex } \\
& S_{i j}=\text { sum of direct and indirect costs of metabolic syndrome- } \\
& \text { related cancer } i \text { in each sex }
\end{aligned}
$$

\section{Direct Costs}

In order to estimate the economic costs of MS-related cancers, a prevalence-based approach was employed that targeted existing and newly diagnosed patients.

MS-related cancers were defined according to the International Classification of Disease 10th revision (ICD-10) codes, and included colorectal cancer (C18-C20), liver cancer (C22), pancreatic cancer (C25), postmenopausal breast cancer (C50), endometrial cancer (uterine corpus) (C54), ovarian cancer (C56) and bladder cancer (C67). Postmenopausal breast cancers were considered as those occurring in patients 50 years of age or older. The target participants of this study were patients who had claimed health insurance benefits with the special cancer claim code (V193) in 2012. In addition, we analyzed patients who had been hospitalized more than once or visited outpatient clinics more than three times [11] with the above mentioned ICD-10 cancer code for sensitivity analysis. Patients under 20 years of age were excluded considering the time lag for MS-related cancer occurrence and lower incidence rates of MSrelated cancers. All calculated costs were converted to US dollars (1 USD = 1127 Korean won) [18].

To measure the direct medical costs, we requested the 2012 data from the Health Insurance Review and Assessment Service. The non-covered medical costs of outpatient and inpatient care were estimated as $16.4 \%$ and $22.8 \%$ of the total direct medical cost [19]. Outpatient pharmaceutical costs were excluded, because they were difficult to calculate and likely accounted for a small portion of the total direct cost [10].

In order to measure transportation costs, we analyzed the raw data contained in the Korea Health Panel-Year Data (version 1.0) of the Korea Institute for Health and Social Affairs, and the average one-way cost among outpatients and inpatients with their guardians was found to be USD 1.60 and USD 11.15, respectively, in 2011. The 2011 price was converted to the 2012 price index by multiplying by the inflation rate.

The cost borne by caregivers was estimated as USD 57.68 per 24 hours by averaging the figures of USD 53.24 per 24 hours for moderate disease and USD 62.11 per 24 hours for serious disease [20]. Inpatients were assumed to be accompanied by caregivers for the entire day, and the costs were multiplied by the number of admissions. For outpatients, the caregivers' cost was estimated as one third of the figure obtained by multiplying the one-day caregivers' cost by the total number of outpatient visits.

The protocol was approved by institutional review board of Korea University (1040548-KU-IRB-13-118-A-1).

\section{Indirect Costs}

Loss of productivity due to premature death is accepted in the human capital approach, and is estimated by calculating the present value of lost future income. The loss of productivity due to premature death for each cancer was calculated using the following formula [21]:

$$
\begin{aligned}
& \sum_{j} \sum_{k m=1}^{n}\left(N_{j k} \times \frac{Y_{j k(t+m)} \times P_{j k(t+m)}}{(1+r)^{m}}\right) \\
& j=\text { sex } \\
& k=\text { age } \\
& m=1,2, \ldots, n \text { ( } n \text { is the difference between age of death and } \\
& \quad \text { life expectancy of the age-cohort) } \\
& t=\text { age at death } \\
& r=\text { discount rate } \\
& N_{j k}=\text { number of deaths from each cancer } \\
& Y_{j k(t+m)}=\text { annual wage income at time }(t+m) \text { by sex and age } \\
& \mathrm{P}_{j k(t+m)}=\text { employment rate at time }(t+m)
\end{aligned}
$$

The number of deaths from each MS-related cancer and figures for annual income by age and sex were obtained from the Korean Statistical Information Service [8] and Statistics of Employment and Labor [22]. A discount rate of 3\% [23] was applied and, for sensitivity analysis, a discount rate of $5 \%[10,11]$ was also applied.

The loss of productivity due to hospitalization was calculated using the days of admission due to each MS-related cancer according to the following formula: 
$\sum D_{j k} \times Y_{j k} \times P_{j k}$

$j=\operatorname{sex}$

$k=$ age

$D_{j k}=$ days for inpatient care due to each cancer

$\mathrm{Y}_{j k}=$ average daily wage income by sex and age

$\mathrm{P}_{j k}=$ employment rate by sex and age

Patients aged between 20 and 69 years who participated in the workforce were included in the calculations for loss of productivity. In order to estimate the loss of productivity due to outpatient visits, one third of the average daily income was multiplied by the number of outpatient visits.

SAS version 9.2 (SAS Institute Inc., Cary, NC, USA) was used for all statistical analyses.

\section{RESULTS}

In 2012, there were 347657 cases of MS-related cancers in Korea, of which 18070 (5.2\%) were attributable to MS (Table 1). The largest proportion of MS-attributable cancers was ac- counted for by postmenopausal breast cancer (6584 cases), followed by colorectal cancer in men. Among age groups, patients aged 50 to 59 years comprised the largest proportion of cases of MS-attributable cancers (6953 patients), followed by patients aged 60 to 69 years (4950 patients).

The economic burden of MS-related cancers in 2012 was USD 3.32 billion, and the burden attributable to MS was USD 199.8 million, comprising $6.02 \%$ of the total cost. Liver cancer in men was associated with the highest cost overall but colorectal cancer in men was associated with the highest economic cost attributable to MS.

The direct cost of cancers attributable to MS in Korean adults aged 20 years and older was estimated to be approximately USD 124.5 million (Table 2). The cost of outpatient care was USD 37.2 million, and the largest proportion of the cost was accounted for by postmenopausal breast cancer (USD 19.5 million), followed by colorectal cancer in men (USD 6.8 million). The estimated cost for the inpatient care of patients attributable to MS was approximately USD 67.8 million, nearly 1.8 times the cost of outpatient care. In inpatient care, the costs for colorectal cancer

Table 1. The number of cases of metabolic syndrome-related cancers attributable to metabolic syndrome

\begin{tabular}{|c|c|c|c|c|c|c|c|c|c|c|}
\hline \multirow{2}{*}{ Cancer site } & \multirow{2}{*}{ Gender } & \multicolumn{8}{|c|}{ Age (y) } & \multirow{2}{*}{ Total } \\
\hline & & $20-29$ & $30-39$ & $40-49$ & $50-59$ & $60-69$ & $70-79$ & $80-89$ & $90+$ & \\
\hline \multirow[t]{2}{*}{ Colon and rectum } & Men & 12 & 87 & 358 & 1042 & 1353 & 1091 & 229 & 12 & 4184 \\
\hline & Women & 14 & 104 & 381 & 937 & 1072 & 1135 & 389 & 27 & 4058 \\
\hline Liver & Men & 1 & 9 & 65 & 186 & 164 & 105 & 20 & 1 & 550 \\
\hline Pancreas & Women & 2 & 10 & 32 & 99 & 150 & 193 & 87 & 8 & 583 \\
\hline Breast, postmenopausal & Women & - & - & - & 3937 & 1772 & 746 & 121 & 8 & 6584 \\
\hline Uterine corpus & Women & 16 & 94 & 240 & 496 & 232 & 74 & 12 & 0 & 1165 \\
\hline Ovary & Women & 34 & 61 & 135 & 195 & 107 & 57 & 11 & 1 & 601 \\
\hline Bladder & Men & 1 & 5 & 19 & 61 & 100 & 119 & 38 & 3 & 346 \\
\hline Subtotal & & 80 & 370 & 1230 & 6953 & 4950 & 3519 & 907 & 61 & 18070 \\
\hline
\end{tabular}

Table 2. Direct costs of cases of metabolic syndrome-related cancers attributable to metabolic syndrome

\begin{tabular}{|c|c|c|c|c|c|c|}
\hline Cancer site & Gender & Outpatient care & Inpatient care & Transportation costs & Caregivers' costs & Total \\
\hline \multirow[t]{2}{*}{ Colon and rectum } & Men & 6751215 & 17315755 & 302513 & 3909621 & 28279104 \\
\hline & Women & 5819308 & 17230783 & 290144 & 4400262 & 27740497 \\
\hline Liver & Men & 1112147 & 4133576 & 42830 & 693289 & 5981842 \\
\hline Pancreas & Women & 1128316 & 5304996 & 62729 & 1270303 & 7766343 \\
\hline Breast, postmenopausal & Women & 19517915 & 15866794 & 519235 & 5888985 & 41792928 \\
\hline Uterine corpus & Women & 1503487 & 2676507 & 61102 & 818156 & 5059252 \\
\hline Ovary & Women & 987023 & 4545684 & 66637 & 1013607 & 6612951 \\
\hline Bladder & Men & 361417 & 686450 & 17525 & 215376 & 1280767 \\
\hline Subtotal (\%) & & 37180826 (29.1) & 67760544 (54.4) & $1362715(1.1)$ & 18209599 (14.6) & $124513684(100)$ \\
\hline
\end{tabular}

Unit: USD. 
Table 3. Indirect costs of cases of metabolic syndrome-related cancers attributable to metabolic syndrome

\begin{tabular}{|c|c|c|c|c|c|c|c|c|}
\hline Cancer site & Gender & $\begin{array}{c}\text { Frequency of } \\
\text { outpatient visits }\end{array}$ & $\begin{array}{l}\text { Loss of productivity } \\
\text { due to outpatient care }\end{array}$ & $\begin{array}{c}\text { Days of } \\
\text { admission }\end{array}$ & $\begin{array}{l}\text { Loss of productivity due } \\
\text { to inpatient admissions }\end{array}$ & $\begin{array}{l}\text { No. of } \\
\text { deaths }\end{array}$ & $\begin{array}{l}\text { Loss of productivity due } \\
\text { to premature death }\end{array}$ & Total \\
\hline \multirow[t]{2}{*}{ Colon and rectum } & Men & 41311 & 933658 & 54901 & 3455587 & 323 & 23165743 & 27554988 \\
\hline & Women & 37049 & 294548 & 64941 & 1222846 & 360 & 7845459 & 9362853 \\
\hline Liver & Men & 5771 & 157533 & 10702 & 814156 & 110 & 12930714 & 13902403 \\
\hline Pancreas & Women & 6783 & 47827 & 20052 & 318073 & 301 & 5576478 & 5942378 \\
\hline $\begin{array}{l}\text { Breast, } \\
\text { postmenopausal }\end{array}$ & Women & 96336 & 1028588 & 71328 & 2276355 & 191 & 7671262 & 10976204 \\
\hline Uterine corpus & Women & 10602 & 136738 & 10837 & 389062 & 48 & 2208244 & 2734043 \\
\hline Ovary & Women & 6805 & 87119 & 15536 & 559350 & 61 & 3455026 & 4101495 \\
\hline Bladder & Men & 2958 & 44695 & 2797 & 104083 & 20 & 565029 & 713807 \\
\hline Subtotal (\%) & & 207615 & 2730705 (3.6) & 251094 & 9139511 (12.1) & 1414 & 63417955 (84.2) & 75288171 \\
\hline
\end{tabular}

Unit: USD.
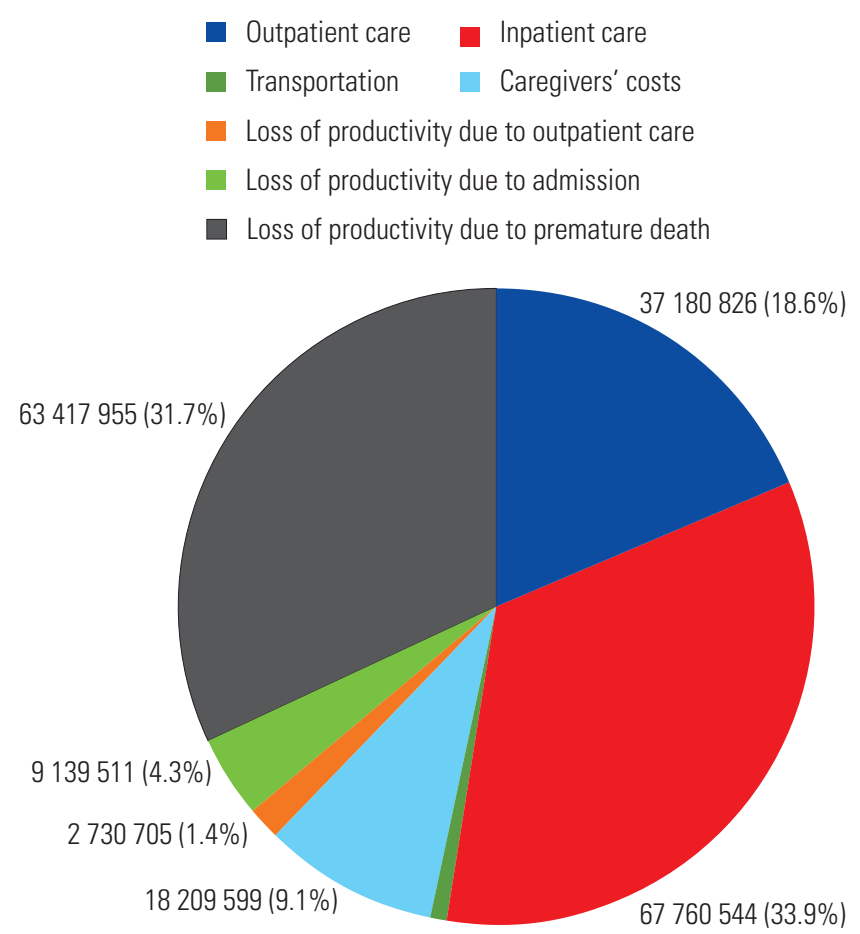

$1362715(0.7 \%)$

Total: 199801855 (100\%)

Figure 1. The economic burden of cancers attributable to metabolic syndrome (unit: USD).

in men and women were USD 17.3 million and USD 17.2 million, respectively, accounting for the two largest proportions of the total cost.

The indirect cost of cancers attributable to MS in Korean adults aged 20 years and older was estimated to be approximately USD 75.3 million (Table 3). The number of premature deaths was 1414 , of which deaths due to colorectal cancer in women made up the largest proportion (360 patients). The estimated losses of productivity due to outpatient care, admission, and premature death were approximately USD 2.7 million, USD 9.1 million, and USD 63.4 million, respectively.

The total economic burden of cancers attributable to MS was approximately USD 199.8 million (Figure 1). By cost item, the costs for inpatient care made up the largest proportion of the total cost (USD 67.8 million), followed by loss of productivity due to premature death (USD 63.4 million). According to cancer site and gender, the largest economic burden was associated with colorectal cancer in men (approximately USD 55.8 million), followed by postmenopausal breast cancer (USD 52.8 million). The economic burden per patient was highest for man liver cancer patients (USD 36 200).

Sensitivity analysis was performed according to the prevalence of MS. The economic burden was found to be USD 242.5 million using the prevalence of MS in 2001 and USD 249.2 million using the prevalence of MS in 2005 (Table 4). In another sensitivity analysis performed according to the patient selection criteria while varying the discount rate using the prevalence of MS in 1998, the economic burden was found to range from at least USD 193.3 million using a discount rate of $5 \%$ to as much as USD 205.2 million using a discount rate of 3\% (Table 5).

\section{DISCUSSION}

This study estimated the economic burden of cancers attributable to MS in Korean adults aged 20 years and older in 2012, and found it to be approximately USD 199.8 million. Several previous studies have addressed the economic burden of various cancers in Korea and other countries, but few studies have 
Table 4. Sensitivity analysis for the prevalence of metabolic syndrome (MS)

\begin{tabular}{llccc}
\hline Year & & $\begin{array}{c}\text { Prevalence of MS } \\
\text { (\%) }\end{array}$ & Direct costs & Indirect costs \\
\hline 1998 & Men & 22.4 & 124513684 & 75288171 \\
& Women & 27.9 & & \\
2001 & Men & 26.9 & 141802168 & 100678723 \\
& Women & 31.8 & & \\
2005 & Men & 31.7 & 142287672 & 106926417 \\
& Women & 29.5 & & \\
\hline
\end{tabular}

Unit: USD.

Table 5. Sensitivity analysis for patient selection criteria and discount rate

\begin{tabular}{|c|c|c|c|}
\hline \multirow[t]{2}{*}{ Selection criteria } & \multirow{2}{*}{$\begin{array}{c}\text { No. of } \\
\text { patients }\end{array}$} & \multicolumn{2}{|c|}{$\begin{array}{l}\text { Economic burden for a } \\
\text { given discount rate }\end{array}$} \\
\hline & & $3 \%$ & $5 \%$ \\
\hline Special code for cancer & 18070 & 199801855 & 193257836 \\
\hline Frequency of visits & 19340 & 205194706 & 198597478 \\
\hline
\end{tabular}

Unit: USD.

investigated the proportion of the economic burden of cancer that is attributable to MS. Since the prevalence of MS and the incidence of MS-related cancers are increasing, analyzing the PAF of MS and estimating economic burden due to MS are of considerable importance for public health. Moreover, the PAF attributable to MS is higher than that of obesity [9] and the economic burden of cancers attributable to MS is much greater than that of cancers due to obesity [24], suggesting that the management of MS is a more urgent policy priority

Meanwhile, because no previous cancer site-specific PAF of MS was available for the Korean population, we calculated a PAF for MS based on the relative risk reported in meta-analyses of data from other countries. This is a limitation of this study, although Korea is becoming westernized rapidly and we modified the PAF of liver cancer to account for factors specific to Korea. The PAFs attributable to MS were calculated for each cancer and varied from $2.2 \%$ of bladder cancer in men to $14.5 \%$ of uterine corpus cancer. In cases where different $R R$ values have been reported, such as colorectal cancer [5,6], 15\% of the difference was reflected in the economic burden varying from USD 78.9 thousand to USD 92.7 thousand according to sensitivity analysis.

In this study, we assumed that MS affects the development of cancers but does not affect the results of the treatment, as reflected by parameters such as survival or death, because the relative risk inherently reflects the occurrence of a condition rather than its outcomes. Therefore, further study of the effects of MS on treatment outcomes is necessary in order to more accurately measure the economic burden attributable to MS.

As we mentioned earlier, we assumed the time lag for cancer occurrence to be 15 or 20 years [9], and conducted sensitivity analysis for the induction period. As a result, the economic burden of cancers attributable to MS in 2012 may have been as high as USD 242.5 million or USD 249.2 million, in order to reflect the increased prevalence of MS in 2001 and 2005, respectively (Table 4). However, the time lag may differ depending on the type of cancer, meaning that applying a single time lag to all types of cancer is another limitation of this study.

We conducted our analysis based on selecting patients with the specific cancer claim code. In Korea, a fee reduction system for medical costs paid by patients with serious diseases such as cancer, stroke, and myocardial infarction has been in place since 2005. We hypothesized that the number of the cancer patients indicated by this code would be more accurate than that calculated using the frequency of visits, because medical institutions providing medical services to cancer patients must claim health insurance benefits using these codes. Sensitivity analysis suggested that a slightly smaller number of patients was selected than would have resulted from a calculation based on frequency of visits, including more than one inpatient admission or more than three outpatient visits (Table 5). Colorectal and breast cancer have a high prevalence in Korea (colorectal cancer, 211 per 100000 people; breast cancer, 175 per 100000 people) [25]. Correspondingly, these cancers accounted for the largest proportions of cases of cancer attributable to MS (postmenopausal breast cancer, 6584 cases; colorectal cancer in men, 4184 cases; colorectal cancer in women, 4058 cases).

In order to estimate the economic burden of MS related-cancers, we utilized the $\mathbf{2 0 1 2}$ data from the Health Insurance Review and Assessment Service. The direct cost was approximately USD 124.5 million, and the costs for outpatient and inpatient care were $29 \%$ and $54 \%$ of this figure, respectively. The costs paid by patients were $4 \%$ and $5 \%$, respectively, which were lower than the $11.8 \%$ figure reported in a previous study [10], because deductibles for the treatment of cancer have decreased from $10 \%$ to $5 \%$ since 2010 . The proportion of transportation costs was also less than that found in previous studies $[10,11]$, due to a higher proportion of ambulatory outpatients with breast cancer. The indirect cost was approximately USD 75.3 million, comprising $37.7 \%$ of the economic burden of cancers 
attributable to MS. This was smaller than the proportion of $76 \%$ reported in a previous study [10] and the proportion of $41.2 \%$ reported for the economic burden of cancer across the European Union [23]. In indirect comparison with foreign countries, as calculated by multiplying the total economic burden of cancers and PAFs, the per capita economic burden of colorectal cancer in Korea was USD 1.86, which was $50 \%$ of that in the US and $75 \%$ of that in the UK $[23,26]$.

The overestimation of productivity loss is a frequently cited limitation of the human capital approach that was employed in our study [21], but this effect was somewhat attenuated in our study due to the lower proportion of frequently fatal cancers such as lung cancer, stomach cancer, and liver cancer. The friction period approach has sometimes been employed as a method of calculating costs [23], but we did not employ this method because it has the potential of underestimating productivity loss and the friction period of Korea has not been studied yet [21].

A few additional limitations characterize our study, one which is the fact that outpatient pharmaceutical costs were excluded. However, the total cost of outpatient anti-cancer drugs multiplied by the PAFs has been estimated to be approximately USD 4.4 million, reflecting $2.19 \%$ of the total economic burden of cancers attributable to MS. It is therefore necessary to calculate more accurate outpatient pharmaceutical costs that are completely matched with each cancer. With regard to transportation costs, the applied average one-way cost generally reflected public transportation fees, potentially underestimating the real cost. The cost of alternative and complementary medicine was not included due to the absence of data.

Despite its limitations, this is the first study on the economic burden of cancers attributable to MS, which is quite significant, since the economic burden of MS has mostly been analyzed in terms of the costs and complications of diabetes and cardiovascular events. While it is important to assess individual risk factors for cancers, such as obesity, the comprehensive evaluation of MS as a risk factor is necessary for the decreasing cancer occurrence.

We found that 18070 cases of cancer were attributable to MS in 2012, and the economic burden was USD 199.8 million. The comprehensive management of MS is necessary to reduce this economic burden, and future studies will help do so by evaluating the occurrence of MS-related cancers in more detail.

\section{ACKNOWLEDGEMENTS}

This study was supported by a grant from the National R\&D Program for Cancer Control, Ministry of Health and Welfare, Korea (grant no. 1320310).

\section{CONFLICT OF INTEREST}

The authors have no conflicts of interest with the material presented in this paper.

\section{REFERENCES}

1. Lim S, Shin H, Song JH, Kwak SH, Kang SM, Won Yoon J, et al. Increasing prevalence of metabolic syndrome in Korea: the Korean National Health and Nutrition Examination Survey for 19982007. Diabetes Care 2011;34(6):1323-1328.

2. Oh SW. Obesity and metabolic syndrome in Korea. Diabetes Metab J 2011;35(6):561-566.

3. Braun S, Bitton-Worms K, LeRoith D. The link between the metabolic syndrome and cancer. Int J Biol Sci 2011;7(7):1003-1015.

4. Bjørge T, Stocks T, Lukanova A, Tretli S, Selmer R, Manjer J, et al. Metabolic syndrome and endometrial carcinoma. Am J Epidemiol 2010;171(8):892-902.

5. Esposito K, Chiodini P, Colao A, Lenzi A, Giugliano D. Metabolic syndrome and risk of cancer: a systematic review and metaanalysis. Diabetes Care 2012;35(11):2402-2411.

6. Esposito K, Chiodini P, Capuano A, Bellastella G, Maiorino MI, Rafaniello C, et al. Colorectal cancer association with metabolic syndrome and its components: a systematic review with metaanalysis. Endocrine 2013;44(3):634-647.

7. National Health Insurance Service. National health insurance statistical yearbook 2012. Seoul: National Health Insurance Service.; 2014, p. 84 (Korean).

8. Korean Statistical Information Service. Death and death rates by cause [cited 2015 Feb 5]. Available from: http://kosis.kr/ wnsearch/totalSearch.jsp (Korean).

9. Park S, Kim Y, Shin HR, Lee B, Shin A, Jung KW, et al. Populationattributable causes of cancer in Korea: obesity and physical inactivity. PLoS One 2014;9(4):e90871.

10. Kim J, Hahm MI, Park EC, Park JH, Park JH, Kim SE, et al. Economic burden of cancer in South Korea for the year 2005. J Prev Med Public Health 2009;42(3):190-198 (Korean).

11. Byun JY, Yoon SJ, Oh IH, Kim YA, Seo HY, Lee YH. Economic burden of colorectal cancer in Korea. J Prev Med Public Health 
2014;47(2):84-93.

12. Häggström C, Rapp K, Stocks T, Manjer J, Bjørge T, Ulmer H, et al. Metabolic factors associated with risk of renal cell carcinoma. PLoS One 2013;8(2):e57475.

13. Ulmer H, Bjørge T, Concin H, Lukanova A, Manjer J, Hallmans G, et al. Metabolic risk factors and cervical cancer in the metabolic syndrome and cancer project (Me-Can). Gynecol Oncol 2012;125(2):330-335.

14. Nagel G, Bjørge T, Stocks T, Manjer J, Hallmans G, Edlinger M, et al. Metabolic risk factors and skin cancer in the Metabolic Syndrome and Cancer Project (Me-Can). Br J Dermatol 2012;167(1): 59-67.

15. Levin ML. The occurrence of lung cancer in man. ActaUniolnt Contra Cancrum 1953;9(3):531-541.

16. Shin A, Park S, Shin HR, Park EH, Park SK, Oh JK, et al. Population attributable fraction of infection-related cancers in Korea. Ann Oncol 2011:22(6):1435-1442.

17. Gold MR, Siegel JE, Russell LB, Weinstein MC. Cost-effectiveness in health and medicine. New York: Oxford University Press; 1996, p. 20.

18. Korea Exchange Bank. The average exchange rate of 2012 [cited 2015 Feb 5]. Available from: http://www.keb.co.kr (Korean).

19. Park M, Choi Y, Tae Y, Choi J, Back S, Lee H. Health insurance patients payments survey, 2010. Seoul: National Health Insurance Corporation; 2011, p.15 (Korean).

20. Jang SC. The subjects for advancement in private employment service. Seoul: Korea Labor Institute; 2013, p. 57 (Korean).

21. Kim Y, Shin S, Park J. Costing methods in healthcare. Seoul: National Evidence-based Healthcare Collaborating Agency; 2013, p.120 (Korean).

22. Ministry of Employment and Labor. Statistics of employment and labor [cited 2015 Feb 5]. Available from: http://laborstat. molab.go.kr (Korean).

23. Luengo-Fernandez R, Leal J, Gray A, Sullivan R. Economic burden of cancer across the European Union: a population-based cost analysis. Lancet Oncol 2013;14(12):1165-1174.

24. Kang JH, Jeong BG, Cho YG, Song HR, Kim KA. Socioeconomic costs of overweight and obesity in Korean adults. J Korean Med Sci 2011;26(12):1533-1540.

25. Jung KW, Won YJ, Kong HJ, Oh CM, Lee DH, Lee JS. Cancer statistics in Korea: incidence, mortality, survival, and prevalence in 2011. Cancer Res Treat 2014;46(2):109-123.

26. Yabroff KR, Lund J, Kepka D, Mariotto A. Economic burden of cancer in the United States: estimates, projections, and future research. Cancer Epidemiol Biomarkers Prev 2011;20(10): 2006-2014. 